A structural decomposition for hypergraphs

Non peer-reviewed author version

Cohen, David A.; Jeavons, Peter G. \& GYSSENS, Marc (1994) A structural decomposition for hypergraphs. In: Barcelo, Hélène; Kalai, Gil (Ed.). Jerusalem Combinatorics '93, p. 161-177.

DOI: $10.1090 /$ conm/178

Handle: http://hdl.handle.net/1942/13464 


\title{
A Structural Decomposition for Hypergraphs
}

\author{
PETER JEAVONS, DAVID COHEN, AND MARC GYSSENS
}

\begin{abstract}
Hypergraphs arise in a variety of applications and are commonly classified as cyclic or acyclic. In this paper we develop a more refined classification scheme for cyclic hypergraphs based on a natural decomposition strategy. The fundamental building blocks in our decompositions are subsets of edges known as $k$-hinges. For any hypergraph, a set of more than $k$ of its edges is defined to be a $k$-hinge if all connected components of the hypergraph with respect to the set of edges meet the latter within at most $k$ of its edges. A $k$-hinge tree is a set of minimal $k$-hinges that cover all edges of $H$, and form a tree with respect to intersection.

The size of the largest node in any 1-hinge tree is shown to be an invariant of the hypergraph, which we call the degree of cyclicity. Acyclic hypergraphs are hypergraphs with degree of cyclicity 2 . The concept of degree of cyclicity was first presented by Gyssens in the context of relational database design, but is presented here for arbitrary hypergraphs with a greatly simplified proof. For more general $k$-hinges we show that it is possible to obtain more powerful decompositions. However, in this case there may be several possible decompositions which do not share any structural invariant. We therefore consider restrictions on $k$-hinges which are necessary in order to guarantee this structural invariant.
\end{abstract}

\section{Introduction}

The complexity of any problem associated with a graph may depend very strongly on the structure of that graph. Examples of such problems are finding a minimal triangulation of a graph [11], coloring problems, and more general constraint satisfaction problems $[\mathbf{6}]$.

Problems which are computationally hard for general graphs may be tractable for particular classes of graphs with some restriction on the structure (for a survey, see $[\mathbf{1}])$. In particular, if the graph may be decomposed into suitable subgraphs (e.g., the biconnected components) in such a way that the problem can be solved for each subgraph separately, and the solutions to these subproblems can

1991 Mathematics Subject Classification. 05C65, 05C85; Secondary 68P15, 68R15, 68T20.

This research was supported by the "British-Flemish Academic Research Collaboration Programme" of the British Council and the Belgian National Fund of Scientific Research.

(C) 0000 American Mathematical Society $0000-0000 / 00 \$ 1.00+\$ .25$ per page 
be efficiently combined to obtain an overall solution, then significant reductions in complexity may be achieved.

The corresponding theory for general hypergraphs is much less well-developed. It has been shown that many problems possess efficient solution techniques when the associated hypergraph is acyclic $[\mathbf{2}, \mathbf{3}]$. However, the classification of hypergraphs into acyclic and cyclic ones is rather crude.

A more powerful decomposition strategy for hypergraphs was developed in the context of a particular application in database theory. This approach led to a hierarchy of classes of hypergraphs in which the acyclic hypergraphs are merely the smallest non-trivial class $[\mathbf{9}, \mathbf{1 0}, \mathbf{7}]$. It has been shown that in the special case of graphs this approach gives rise to a finer decomposition than the decomposition into biconnected components, and therefore provides a more informative bound on the complexity of many problems $[\mathbf{7}, \mathbf{8}]$.

In this paper, we develop a very general decomposition strategy for hypergraphs in the context of general hypergraph theory and discuss its properties. The paper is organized as follows. In Section 2, we introduce some terminology and define hypergraph decompositions in terms of $k$-hinges and $k$-hinge-trees. In Section 3, we review the desirable properties of 1-hinges and 1-hinge-trees that motivated this study and propose a new, short, and more insightful proof of the main property. The remaining sections are devoted to general $k$-hinges. In Section 4, we demonstrate the usefulness of general $k$-hinge-tree decompositions. In Section 5 we discuss the difficulties that arise when trying to build a general theory for constructing arbitrary $k$-hinge-tree decompositions, and the open problems resulting from them. Most of the difficulties arise from the fact that the desirable properties for 1-hinges exhibited in Section 3 do not carry over to arbitrary $k$-hinges, even for $k=2$.

\section{Definitions}

The building blocks of the decomposition we intend to propose are so-called $k$-hinges, which are defined in this section. Our notion of hypergraph decomposition is then formalized by the concept of $k$-hinge-trees.

First though, we briefly review some of the relevant terminology concerning hypergraphs.

DEFINITION 2.1. [4] A hypergraph is an ordered pair $(V, E)$ where $V$ is a finite set of vertices and $E$ is a set of edges, each of which is a subset of $V$.

Undirected graphs can be seen as special cases of hypergraphs, where each edge contains exactly two vertices.

DEFinition 2.2. Let $(V, E)$ be a hypergraph, let $H \subseteq E$, and let $F \subseteq E-H$. $F$ is called connected with respect to $H$ if, for any two edges $e, f \in F$, there exists a sequence $e_{1}, \ldots, e_{n}$ of edges in $F$ such that (i) $e_{1}=e$; (ii) for $i=1, \ldots, n-1$, $e_{i} \cap e_{i+1} \nsubseteq \bigcup H$; and (iii) $e_{n}=f$. Such a sequence is called a path with respect to $H$ connecting $e$ and $f$ in $F$. 
The maximal connected subsets of $E-H$ with respect to $H$ are called the connected components of $E-H$ with respect to $H$. They obviously form a partition of $E-H$. In the case that $H$ is the empty set of edges, we usually drop the phrase "with respect to $H$ " and simply speak about connected subsets and connected components of $E$. If $E$ itself is connected, then $(V, E)$ is said to be a connected hypergraph. In this paper, we shall assume that all hypergraphs to be decomposed are connected, since in a disconnected hypergraph each connected component may be decomposed individually.

Using this notion of connectivity we now define the sets of edges which will be called $k$-hinges:

Definition 2.3. Let $(V, E)$ be a hypergraph, and let $H$ be either $E$ or a proper subset of $E$ containing at least $k+1$ edges. Let $H_{1}, \ldots, H_{m}$ be the connected components of $E-H$ with respect to $H$. Then $H$ is called a $k$-hinge if, for $i=1, \ldots, m$, there exists a set $h_{i} \subseteq H$ consisting of $k$ edges such that

$$
\left(\bigcup H_{i}\right) \cap(\bigcup H) \subseteq \bigcup h_{i} .
$$

The set of edges $h_{i}$ is called a separating edge set for $H_{i}$.

In words, a $k$-hinge of a hypergraph is either the entire hypergraph or a subset $H$ of its edges (containing at least $k+1$ edges) with the property that the set of nodes in each connected component with respect to that subset intersects the set of nodes in $H$ within at most $k$ edges.

In general, $k$-hinges can in turn contain other $k$-hinges. We are most interested in $k$-hinges that do not contain other $k$-hinges; these are called minimal $k$-hinges.

Our aim is to decompose any hypergraph into a "tree" of minimal $k$-hinges, with the following properties:

Definition 2.4. Let $(V, E)$ be any hypergraph. A $k$-hinge-tree of $(V, E)$ is a tree $^{1},(N, A)$, with nodes $N$ and labeled $\operatorname{arcs}^{2} A$, such that

(i) each node in $N$ is a minimal $k$-hinge of $(V, E)$, and each label of an arc in $A$ is a subset of $E$;

(ii) $\bigcup N=E$;

(iii) for each labeled arc $\left(\left\{n_{i}, n_{j}\right\}, E^{\prime}\right) \in A, E^{\prime}=n_{i} \cap n_{j}$ and satisfies $\left(\bigcup n_{i}\right) \cap$ $\left(\bigcup n_{j}\right)=\bigcup E^{\prime}$ and $1 \leq\left|E^{\prime}\right| \leq k$; and

(iv) the vertices of $V$ shared by two tree nodes are entirely contained within each tree node on their connecting path.

Property 3 states that adjacent tree nodes share at most $k$ edges of $E$, and this set of edges is also the label of their connecting tree-arc; moreover, the vertices shared by the adjacent tree nodes are precisely the vertices of this edge set.

Note that a hypergraph may, in general, have many different $k$-hinge-trees, containing different sets of minimal $k$-hinges.

Below, we give an example of a $k$-hinge-tree of a hypergraph for $k=1$.

\footnotetext{
${ }^{1}$ By "tree", we understand an unrooted tree, i.e., an undirected acyclic graph.

${ }^{2} \mathrm{~A}$ labeled arc is formally defined as an ordered pair $\left(\left\{n_{i}, n_{j}\right\}, a\right)$ with $n_{i}$ and $n_{j}$ different tree nodes and $a$ the arc-label.
} 


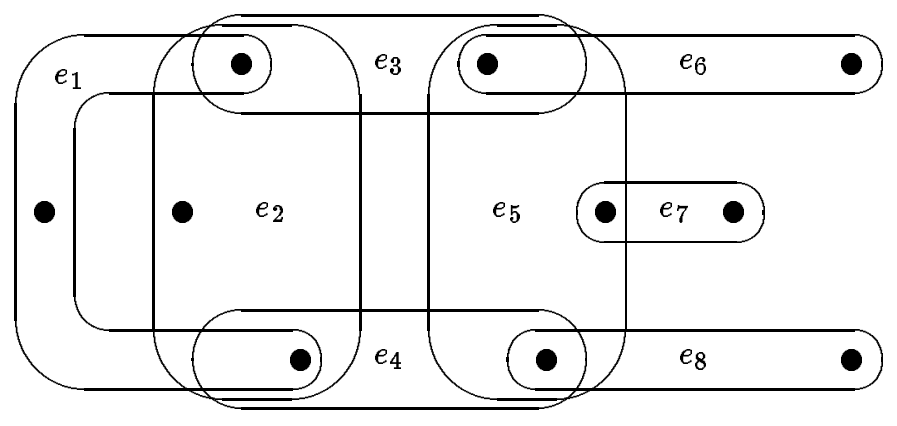

Figure 1. A hypergraph (Example 2.1).

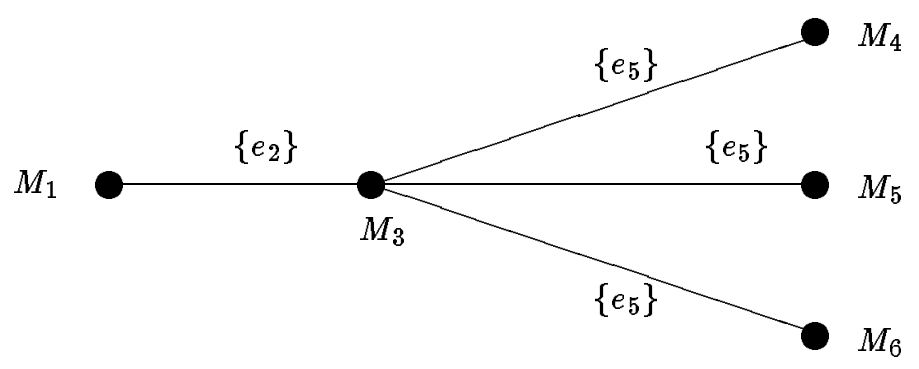

FIGURE 2. A 1-hinge-tree of the hypergraph in Figure 1.

Example 2.1. Consider the hypergraph illustrated in Figure 1. The minimal 1-hinges of the hypergraph in Figure 1 are the following:

$$
\begin{array}{ll}
M_{1}=\left\{e_{1}, e_{2}\right\} ; & M_{4}=\left\{e_{5}, e_{6}\right\} \\
M_{2}=\left\{e_{1}, e_{3}, e_{4}, e_{5}\right\} ; & M_{5}=\left\{e_{5}, e_{7}\right\} \\
M_{3}=\left\{e_{2}, e_{3}, e_{4}, e_{5}\right\} ; & M_{6}=\left\{e_{5}, e_{8}\right\}
\end{array}
$$

Figure 2 illustrates one possible 1-hinge-tree of the hypergraph in Figure 1.

An alternative 1-hinge-tree of $(V, E)$ may be obtained by replacing $M_{3}$ with $M_{2}$ and labeling the arc connecting it to $M_{1}$ with $\left\{e_{1}\right\}$.

Definition 2.4 is partly inspired by the notion of tree developed by Courcelle [5] in the context of (binary) graphs, and is a generalization of the notion of "hingetree" in $[\mathbf{7}, \mathbf{8}]$ (which correspond to 1-hinge-trees in this paper). The introduction of general $k$-hinge-trees was motivated by the observation that, while 1-hinge-tree decompositions have many desirable properties with respect to solving problems defined on a hypergraph, many hypergraphs do not possess non-trivial 1-hingetree decompositions but do possess interesting $k$-hinge tree decompositions for higher values of $k$. 


\section{Decompositions using 1-hinges}

The concept of 1-hinge was introduced in [9] under the name "hinge" in the context of relational database theory. It was subsequently found that every hypergraph has at least one 1-hinge-tree, and that the size of the largest node in any 1-hinge-tree decomposition is an invariant of the hypergraph, i.e., independent of the particular 1 -hinge-tree chosen [7]. This result has subsequently been applied to the analysis of constraint satisfaction problems [8].

Unfortunately, the initial proof in [7] heavily depends on properties of certain database concepts and therefore is not suitable for possible generalization to decomposition into arbitrary $k$-hinge-trees. Therefore, we exhibit a simpler proof stated entirely within the context of general hypergraph theory.

In Section 3.1, we describe two key properties of 1-hinges. Using these key properties, we then prove the actual invariance result in Section 3.2. For completeness, an algorithm to find a particular 1-hinge-tree for any hypergraph is given in Section 3.3. Because of the invariance result, the particular 1-hinge-tree found by this algorithm is optimal.

3.1. Properties of 1 -hinges. The first of the two key properties of 1-hinges we shall exhibit in this section was originally proved in [9] by a straightforward consideration of the possible connected components with respect to $H^{\prime}$ and will here be referred to as the "Inheritance Property:"

Inheritance Property. [9] Let $(V, E)$ be a hypergraph, let $H$ be a 1-hinge of $(V, E)$, and let $H^{\prime} \subseteq H$. Then $H^{\prime}$ is a 1-hinge of $(V, E)$ if and only if it is a 1-hinge of the hypergraph $(\bigcup H, H)$.

To state the second property, which we shall call the "Decomposition Property," we first propose a notion of decomposition in Definition 3.1. With a possible generalization in mind, we state Definition 3.1 in a slightly more general fashion than strictly needed in this section.

DEFinition 3.1. Let $(V, E)$ be a hypergraph, let $H$ be a subset of edges, and let $h$ be a subset of $E$. An $h$-decomposition of $H$ is any set, $\mathcal{D}$, of subsets of $H$, such that $H=\bigcup \mathcal{D}$ and, for all $S, T \in \mathcal{D}, S \neq T,(\bigcup S) \cap(\bigcup T) \subseteq \bigcup h$.

Decomposition Property. Let $(V, E)$ be a hypergraph, and let $H$ be a 1-hinge of $(V, E)$. Let $\epsilon$ be an arbitrary edge in $E$, and let $\mathcal{D}$ be an $\{e\}-$ decomposition of $H$. There exists $S \in \mathcal{D}$ such that, for every $\mathcal{D}^{\prime} \subseteq \mathcal{D}$ with $S \in \mathcal{D}^{\prime}, \bigcup \mathcal{D}^{\prime}$ is either a 1-hinge of $(V, E)$ or a single edge in $E$.

Proof. If $e \notin H$, let $C_{e}$ be the connected component of $E-H$ with respect to $H$ which contains $e$, and let $\{f\}$ be a corresponding singleton separating edge set in $H$. If $e \in H$, let $C_{e}=\emptyset$, and let $f=e$. In either case, let $S$ be an element of $\mathcal{D}$ containing $f$. Let $H^{\prime}=H \cup C_{e}$. Note that, as $H^{\prime}$ is a 1-hinge of $(V, E)$, every 1-hinge of $\left(\bigcup H^{\prime}, H^{\prime}\right)$ is also a 1-hinge of $(V, E)$, by the Inheritance Property. 
We have

$$
\begin{aligned}
\left(\bigcup \mathcal{D}^{\prime}\right) \cap(\bigcup & \left.\left(H^{\prime}-\left(\bigcup \mathcal{D}^{\prime}\right)\right)\right) \\
& =\left[\left(\bigcup \mathcal{D}^{\prime}\right) \cap\left(\bigcup\left(H^{\prime}-H\right)\right)\right] \cup\left[\left(\bigcup \mathcal{D}^{\prime}\right) \cap\left(\bigcup\left(H-\left(\bigcup \mathcal{D}^{\prime}\right)\right)\right)\right] \\
& \subseteq\left[(\bigcup H) \cap\left(\bigcup C_{e}\right)\right] \cup\left[\left(\bigcup \mathcal{D}^{\prime}\right) \cap\left(\bigcup\left(H-\left(\bigcup \mathcal{D}^{\prime}\right)\right)\right) \cap(\bigcup H)\right] \\
& \subseteq f \cup(e \cap(\bigcup H)) \\
& \subseteq f
\end{aligned}
$$

Hence if $S \in \mathcal{D}^{\prime}$, then $\bigcup \mathcal{D}^{\prime}$ is either $\{f\}$ or a 1-hinge of $\left(\bigcup H^{\prime}, H^{\prime}\right)$, in which case it is a 1-hinge of $(V, E)$.

The Decomposition Property has the following immediate corollary:

Inseparability Property. Let $(V, E)$ be a hypergraph and let $H$ be a minimal 1-hinge of $(V, E)$. Let $e$ be an arbitrary edge in $E$ and let $\mathcal{D}$ be an $\{e\}-$ decomposition of $H$. Then $\mathcal{D}$ has at most two nonempty elements, and at most one element containing more than one edge.

3.2. An invariant of 1 -hinge-trees. Using the properties described above, we can now prove the following:

Proposition 3.1. Let $(V, E)$ be a hypergraph and let $T=(N, A)$ be a 1-hingetree for $(V, E)$. For any minimal 1-hinge $H$ of $(V, E),|H| \leq \max \{|n| \mid n \in N\}$.

Proof. Every arc of $T$ divides $T$ into two subtrees, and by Properties 3 and 4 in Definition 2.4, the partition of $H$ defined by these subtrees is an $\{e\}$-decomposition of $H$. By the minimality of $H$ and the Inseparability Property, one of the two subsets in this partition contains at most one edge of $H$. Hence we may orient each arc of $T$ towards a subtree containing at most one edge of $H .^{3}$

As $T$ is now a directed tree, there is some node $n_{c}$ with in-degree zero. By the choice of orientation, each branch out of $n_{c}$ contains at most one edge of $H .^{4}$

If there are two branches out of $n_{c}$ both labeled with the same edge $\{e\}$ and each containing a distinct single edge of $H$, then we have an $\{e\}$-decomposition of $H$ containing two singleton sets, so the Inseparability Property implies that $|H|=2$, whence the result holds trivially.

Otherwise, all branches out of $n_{c}$ labeled with the same edge contain at most one edge of $H$ between them. By Property 3 of Definition 2.4, if $\{e\}$ is the label of the arc connecting a branch out of $n_{c}$ to $n_{c}$, then $e$ is the only edge contained in both $n_{c}$ and the branch. Hence, if $e \notin H$ and if the branches connected to $n_{c}$ by arcs labeled $\{e\}$ do contain an edge of $H$ between them, then that edge cannot be contained in $n_{c}$. Let $L$ denote the set of edges that is the union of the labels of the arcs connecting branches to $n_{c}$. By the preceding argument, it

\footnotetext{
${ }^{3}$ When we say that a subtree contains an edge we mean that that subtree has a node containing that edge.

${ }^{4}$ The branches are defined not to contain the central node.
} 


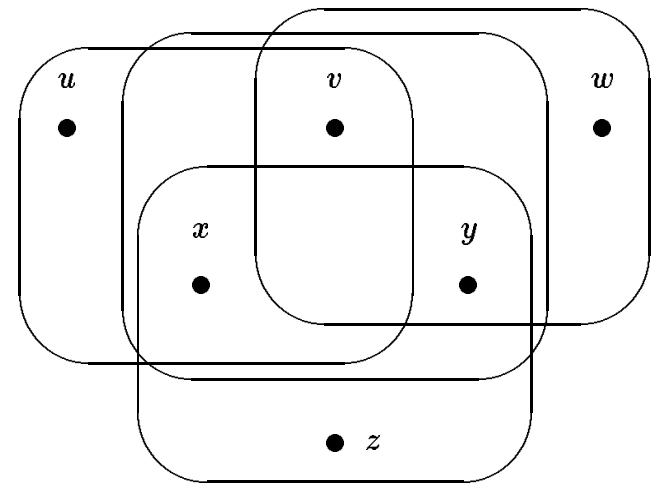

Figure 3. The hypergraph $\left(V, E^{\prime}\right)$ (Example 3.1).

follows that $|L-H| \geq\left|H-n_{c}\right|$. Since $L \subseteq n_{c},\left|n_{c}-L\right| \geq\left|H-n_{c}\right|$, whence $\left|n_{c}-H\right| \geq\left|H-n_{c}\right|$, whence $\left|n_{c}\right| \geq|H|$, whence the theorem.

Invariance Theorem. For any hypergraph, $(V, E)$, the size of the largest node in a 1-hinge-tree of $(V, E)$ is an invariant.

Proof. Let $(V, E)$ be a hypergraph, and let $T$ be any 1-hinge-tree of $(V, E)$. By Proposition 3.1, the size of the largest node in $T$ is equal to the size of the largest minimal 1-hinge of $(V, E)$.

The size of the largest minimal 1-hinge of a hypergraph will be referred to as the degree of cyclicity. A hypergraph will be called $n$-cyclic if its degree of cyclicity is less than or equal to $n$. Acyclicity [2] is equivalent to 2-cyclicity [7].

Example 3.1. First consider the hypergraph in Figure 1, Example 2.1. As the largest node in the 1-hinge-tree illustrated in Figure 2 has size 4, the hypergraph is 4-cyclic. Notice that the largest node of the other 1-hinge-tree described in Example 1 also has size 4, in accordance with the Invariance Theorem.

Next, consider the hypergraph $(V, E)$ with 6 vertices, $u, v, w, x, y$, and $z$, and 3 edges, $\{x, y, z\},\{v, w, y\}$, and $\{u, v, x\}$. The only 1-hinge of $(V, E)$ is the set of all 3 edges, so $(V, E)$ is 3 -cyclic (and $n$-cyclic for all $n \geq 3$ ). If we add an additional edge, $\{v, x, y\}$, we obtain a new hypergraph, $\left(V, E^{\prime}\right)$, as shown in Figure 3.

The hypergraph $\left(V, E^{\prime}\right)$ contains three 1-hinges of size 2 :

$$
\{\{v, x, y\},\{u, v, x\}\} ;\{\{v, x, y\},\{v, w, y\}\} ; \text { and }\{\{v, x, y\},\{x, y, z\}\} \text {. }
$$

Any tree with these three nodes and with arcs labeled $\{v, x, y\}$, is a 1-hinge-tree of $\left(V, E^{\prime}\right)$, showing that $\left(V, E^{\prime}\right)$ is 2-cyclic (i.e., acyclic).

This example illustrates the surprising fact that the addition of an edge may reduce the degree of cyclicity, and may even turn a cyclic hypergraph into an acyclic hypergraph. 
3.3. An algorithm for 1-hinge-trees. Given a hypergraph $(V, E)$, a 1hinge-tree may be obtained in polynomial time by the following algorithm $[8]$ :

Hinge-Tree Decomposition Algorithm.

Input: A hypergraph $(V, E)$.

Output: A 1-hinge-tree $T$ for $(V, E)$.

Method:

(i) Mark each edge in $E$ as unused. Set $i=0, N_{0}=\{E\}$ and $A_{0}=\emptyset$, and mark the node $E$ in $N_{0}$ as non-minimal.

(ii) If all nodes of $N_{i}$ are marked minimal, then set $T=\left(N_{i}, A_{i}\right)$ and stop. Else, choose a non-minimal node $F$ in $N_{i}$.

(iii) If all edges in $F$ are marked used, then mark $F$ as minimal and return to 2. Else, choose an unused edge $e \in F$ and mark $e$ as used.

(iv) Let $\Gamma=\{G \cup\{e\} \mid G$ is a connected component of $F-\{e\}$ w.r.t. $e\}$, and let $\gamma: F \rightarrow \Gamma$ be any function such that for all $f \in F, f \in \gamma(f)$. If $|\Gamma|=1$, then return to 3 .

(v) Set $N_{i+1}=\left(N_{i}-\{F\}\right) \cup \Gamma$ and

$$
\begin{aligned}
A_{i+1}=\left(A_{i}-\left\{\left(\left\{F, F^{\prime}\right\}\right.\right.\right. & \left.\left.,\{f\}) \mid\left(\left\{F, F^{\prime}\right\},\{f\}\right) \in A_{i}\right\}\right) \\
& \cup\left\{\left(\left\{\gamma(f), F^{\prime}\right\},\{f\}\right) \mid\left(\left\{F, F^{\prime}\right\},\{f\}\right) \in A_{i}\right\} \\
& \cup\{(\{\gamma(f), \gamma(e)\},\{e\}) \mid f \in F, \gamma(f) \neq \gamma(e)\},
\end{aligned}
$$

and mark all the new nodes added to $N_{i+1}$ as non-minimal.

(vi) Increment $i$ and return to 2 .

The correctness of the algorithm relies on the Inheritance Property, the Decomposition Property, and the Inseparability Property.

At Step 3, we always have a set of nodes $N_{i}$ which form a tree of 1-hinges, but which are not always minimal 1-hinges. At Step 4, we examine each edge $e$ of $F$ to see if it gives rise to an $\{e\}$-decomposition of $F-\{e\}$ with two or more non-empty elements. By the Decomposition Property, the existence of such a decomposition means that the node is non-minimal, so it is decomposed into smaller nodes. The fact that these smaller nodes are 1-hinges of the original hypergraph follows from the Inheritance Property. If no edge separates a node in this way then each node of $N_{i}$ is minimal so we have a valid 1-hinge-tree and the algorithm stops.

\section{Decompositions using arbitrary $k$-hinges}

In the previous section, we have shown that there is a straightforward decomposition strategy using 1-hinges, which provides a useful measure for the structural complexity of hypergraphs. However, the minimal 1-hinges may be quite large in many cases, so it is natural to look for more powerful decompo- 


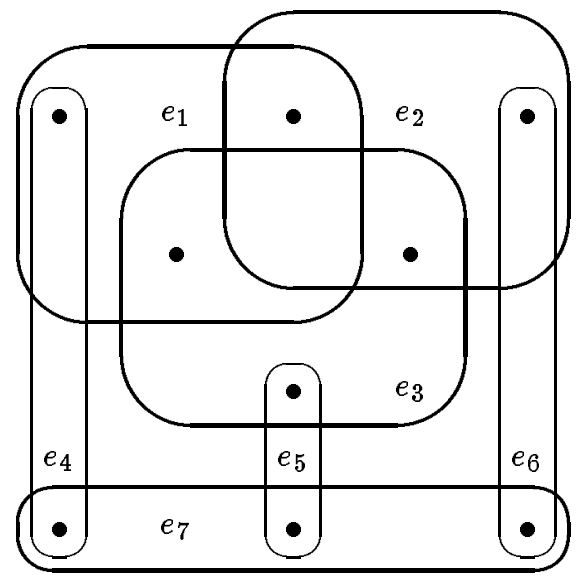

FiguRE 4. A hypergraph with a 2-hinge-tree decomposition.

sition strategies which can break down hypergraphs into smaller units. In this section, we show that more powerful decompositions are possible in certain cases if we are not restricted to 1-hinges.

The following example shows that a hypergraph may have a useful 2-hinge-tree decomposition even when it contains no proper 1-hinges:

EXAMPLE 4.1. Figure 4 shows a hypergraph with 7 edges, $e_{1}, \ldots, e_{7}$, containing no smaller 1-hinges. It therefore has degree of cyclicity 7 . However, this hypergraph has a 2-hinge-tree in which the size of the largest node is 4 . The largest 2-hinge in this tree, $\left\{e_{1}, \epsilon_{2}, e_{3}, e_{7}\right\}$, is indicated by the heavy lines.

Example 4.1 also shows that minimal 2-hinges need not be connected, in contrast to minimal 1-hinges which are always connected $[\mathbf{9}]$.

The next result demonstrates the power of 2-hinges for the decomposition of series-parallel graphs:

Proposition 4.1. Any series-parallel graph has a 2-hinge-tree decomposition in which every tree node contains at most three edges.

Proof. Let $G=(V, E)$ be a series-parallel graph with terminals $u$ and $v$. For any such graph we may write $G=G_{1} * G_{2}$, where $G_{1}=\left(V_{1}, E_{1}\right), G_{2}=\left(V_{2}, E_{2}\right)$, and "*" represents either series combination or parallel combination. Choose any edges $\epsilon_{1} \in E_{1}$ and $e_{2} \in E_{2}$ such that $u \in \epsilon_{1}$ and $v \in e_{2}$. We will prove, by induction on the number of edges, that $G$ can be decomposed into a 2-hinge-tree in which the largest node has size 3 , and some node contains both $e_{1}$ and $e_{2}$.

If $|E| \leq 3$, then $E$ itself, obviously containing $e_{1}$ and $e_{2}$, is a minimal 2-hinge, yielding a single-node 2 -hinge-tree, so the result holds trivially. Now assume that $|E|>3$ and that the induction hypothesis holds for all series-parallel graphs with fewer than $|E|$ edges. The graph $G$ may be written as $G_{1} * G_{2}$, as above, with $\left|E_{1}\right|<|E|$ and $\left|E_{2}\right|<|E|$. Let the terminals of $G_{1}$ be $u_{1}$ and $v_{1}$, and let the terminals of $G_{2}$ be $u_{2}$ and $v_{2}$. Choose $f_{1} \in E_{1}$ and $f_{2} \in E_{2}$ such that $u_{1} \in e_{1}$ 
and $v_{1} \in f_{1}$ or vice versa and $u_{2} \in e_{2}$ and $v_{2} \in f_{2}$ or vice versa. If possible, $f_{1}$ should be chosen different from $e_{1}$, and $f_{2}$ different from $e_{2}$. By the induction hypothesis, there exists a 2-hinge-tree $T_{1}^{\prime}$ of $G_{1}$ with a node $n_{1}^{\prime}$ containing both $e_{1}$ and $f_{1}$, and a 2-hinge-tree of $G_{2}$ with a node $n_{2}^{\prime}$ containing both $e_{2}$ and $f_{2}$. Let $n_{1}=\left\{e_{1}, e_{2}, f_{1}\right\}$, and $n_{2}=\left\{e_{1}, e_{2}, f_{2}\right\}$. Then $n_{1}$ (respectively $n_{2}$ ) is a 2-hinge of $G$ if its size is 3 . There are three cases to consider:

Case 1. $\left|E_{1}\right|=1$ or $\left|E_{2}\right|=1$. We only consider the first possibility. From $\left|E_{1}\right|=1$ and $|E|>3$ it follows that $\left|E_{2}\right|>2$. By the choice of $f_{2}$, it then follows that $\left|n_{2}\right|=3$, whence $n_{2}$ is a 2-hinge of $G$. It can also be seen straightforwardly that all nodes of $T_{2}^{\prime}$ are also minimal 2-hinges of $G$. A 2-hinge-tree of $G$ with a node containing both $e_{1}$ and $e_{2}$ is now obtained from $T_{2}^{\prime}$ and $n_{2}$ by connecting $n_{2}$ to $n_{2}^{\prime}$, and labeling the arc $\left\{\epsilon_{2}, f_{2}\right\}$.

Case 2. $\left|E_{1}\right|=2$ or $\left|E_{2}\right|=2$. Again, we only consider the first possibility. From $\left|E_{1}\right|=2$, it follows that $\left|E_{2}\right| \geq 2$, whence both $\left|n_{1}\right|=3$ and $\left|n_{2}\right|=3$, and both $n_{1}$ and $n_{2}$ are 2-hinges of $G$. Now if $\left|E_{2}\right|=2$, a 2-hinge-tree of $G$ with a node containing both $e_{1}$ and $e_{2}$ is obtained from $n_{1}$ and $n_{2}$ by simply connecting them, and labeling the arc $\left\{e_{1}, \epsilon_{2}\right\}$. If $\left|E_{2}\right|>2$, all nodes of $T_{2}^{\prime}$ are also minimal 2-hinges of $G$, as in Case 1. A 2-hinge-tree of $G$ with a node containing both $e_{1}$ and $e_{2}$ is now obtained from $T_{2}^{\prime}, n_{1}$, and $n_{2}$ by connecting $n_{1}$ to $n_{2}, n_{2}$ to $n_{2}^{\prime}$, and labeling the arcs appropriately.

Case 3. $\left|E_{1}\right|>2$ and $\left|E_{2}\right|>2$. Then both $\left|n_{1}\right|=3$ and $\left|n_{2}\right|=3$, both $n_{1}$ and $n_{2}$ are 2-hinges of $G$, and both the nodes of $T_{1}^{\prime}$ and $T_{2}^{\prime}$ are minimal 2-hinges of $G$. A 2-hinge-tree for $G$ with a node containing both $e_{1}$ and $e_{2}$ is now obtained from $T_{1}^{\prime}, T_{2}^{\prime}, n_{1}$, and $n_{2}$ by connecting $n_{1}$ to $n_{2}, n_{1}$ to $n_{1}^{\prime}$, and $n_{2}$ to $n_{2}^{\prime}$, and labeling the arcs appropriately.

We can apply Proposition 4.1 to obtain a decomposition for the cycle graph. (Note that in the cycle graph every set of three edges is a 2-hinge.)

Example 4.2 . The cycle graph $C_{n}$ with $n$ vertices and edges, $n \geq 3$, has a 2-hinge-tree where every node contains exactly three edges. This tree is in fact a linear list. We construct it by consecutively labeling the edges as $0,1, \ldots n-1$ and putting $N_{i}=\{\{0, i, i+1\} \mid i=1, \ldots, n-2\}$. The required 2-hinge-tree is now obtained by connecting sets of edges with consecutive indices.

The next result shows that most graphs contain many small $k$-hinges:

Proposition 4.2. In any graph, every set of $k+1$ edges containing a path of length 3 is a $k$-hinge.

Proof. Let $G=(V, E)$ be a graph, and let $E^{\prime}=\left\{e_{1}, e_{2}, \ldots, e_{k+1}\right\}$ be a subset of edges containing a path of length 3 . Without loss of generality, assume that $e_{1}, e_{2}, e_{3}$ form a path. Then $\bigcup E^{\prime}=\bigcup\left(E^{\prime}-e_{2}\right)$. Hence $E^{\prime}-e_{2}$ is a separating set of $k$ edges for each connected component of $E-E^{\prime}$ with respect to $E^{\prime}$, whence $E^{\prime}$ is a $k$-hinge. 
Since there are so many $k$-hinges with $k+1$ edges in simple graphs, it is natural to ask whether a minimal $k$-hinge in a graph can be very large. We therefore list some examples of large minimal 2-hinges in graphs.

Example 4.3 .

(i) Any 1-factor of $K_{2 n}$ is a minimal 2-hinge with $n$ edges. ${ }^{5}$

(ii) Any maximal star, $K_{1, n-1}$, in $K_{n}, n \geq 4$, is a connected minimal 2-hinge with $n-1$ edges. ${ }^{6}$

(iii) Consider the previous example. If each edge of $K_{n}$ not in $K_{1, n-1}$ is replaced by a path of length $2, K_{1, n-1}$ remains a connected minimal 2-hinge with $n-1$ edges which is moreover vertex-generated.

In order to decompose an arbitrary graph into a $k$-hinge-tree we are sometimes forced to use $k$-hinges which contain more than $k+1$ edges, as the following proposition shows:

Proposition 4.3. There is no $k$-hinge-tree of $K_{2 k+3}$ in which every node has size $k+1$.

Proof. Assume, for contradiction, that there exists a $k$-hinge-tree $T$ of $K_{2 k+3}$ in which every node has size $k+1$. Thus each node of $T$ contains at most $2 k+2$ vertices. Let $n_{1}$ be a node in $T$ of which the number of vertices is maximal. Let $v_{2}$ be an arbitrary vertex of $K_{2 k+3}$ not in $n_{1}$ (such a vertex exists), and let $n_{2}$ be a node of $T$ containing $v_{2}$ such that the distance in $T$ between $n_{1}$ and $n_{2}$ is minimal. By the choice of $n_{1}$ and $n_{2}, n_{1}$ contains a vertex, say $v_{1}$, not in $n_{2}$. Let $n_{3}$ be an arbitrary node of $T$ containing the edge $\left\{v_{1}, v_{2}\right\}$. Clearly, $n_{3} \neq n_{1}$ and $n_{3} \neq n_{2}$. By Property 4 of Definition 2.4, the node $n_{2}$ is not on the path in $T$ connecting $n_{1}$ and $n_{3}$, since $n_{1}$ and $n_{3}$ share the vertex $v_{1}$, which is not in $n_{2}$. Similarly, $n_{1}$ cannot be on the path connecting $n_{2}$ and $n_{3}$. Hence the path connecting $n_{1}$ to $n_{2}$ contains an internal node $n_{4}$ which is both on the path connecting $n_{1}$ to $n_{3}$ and the path connecting $n_{2}$ to $n_{3} \cdot{ }^{7}$ By Property 4 of Definition 2.4, the node $n_{4}$ contains the vertex $v_{2}$ shared by the nodes $n_{1}$ and $n_{3}$. However $n_{4}$ is closer to $n_{1}$ than is $n_{2}$, a contradiction. Hence $K_{2 k+3}$ has no $k$-tree in which every node has size $k+1$.

\section{Difficulties and open problems for arbitrary $k$-hinges}

Since arbitrary $k$-hinges allow more powerful decompositions than 1-hinges, it is natural to ask whether the results of Section 3 can be generalized to $k$-hinges. In other words, is it also true that, for $k>1$, every hypergraph has a $k$-hinge-tree decomposition, and if so, is there an associated invariant complexity measure $\Gamma$

\footnotetext{
${ }^{5} \mathrm{~A} 1$-factor of a graph is a set of edges that are pairwise non-incident and cover the set of nodes. For any number $n, K_{n}$ denotes the complete graph on $n$ vertices.

${ }^{6} \mathrm{~A}$ star is a graph the edges of which are incident with a common vertex.

${ }^{7}$ Observe that $n_{3}$ and $n_{4}$ can be equal.
} 


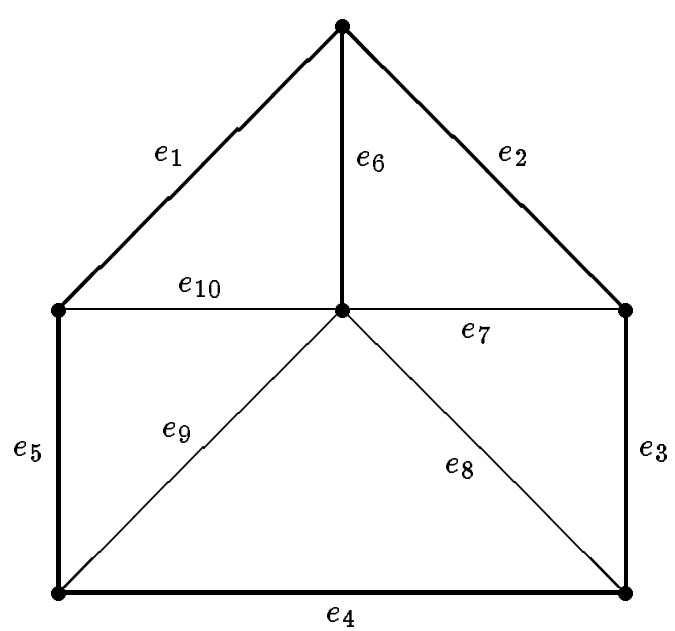

Figure 5. A hypergraph illustrating that 2-hinges do not have the Inheritance Property.

In order to establish the results for 1-hinges we made use of the Inheritance Property, the Decomposition Property, and the Inseparability Property. The following results indicate that these properties do not directly generalize to arbitrary $k$-hinges.

First of all unlike 1-hinges, 2-hinges do not have the Inheritance Property.

ExAMPLE 5.1. Figure 5 shows a graph, $G$, with edges $e_{1}, \ldots, e_{10}$, which contains a 2-hinge, $H=\left\{\epsilon_{1}, \ldots, \epsilon_{5}, e_{6}\right\}$, indicated by heavy lines. The subgraph $(\bigcup H, H)$ contains a 2-hinge, $H^{\prime}=\left\{\epsilon_{1}, \ldots, \epsilon_{5}\right\}$, which is not a 2-hinge of $G$.

Furthermore, 2-hinges do not have the Decomposition Property, the obvious generalization of which to arbitrary $k$-hinges is as follows:

Decomposition Property for $k$-Hinges. Let $(V, E)$ be a hypergraph and let $H$ be a $k$-hinge of $(V, E)$. Then $H$ satisfies the decomposition property if for every subset $h \subseteq E$ containing $k$ edges and for every $h$-decomposition $\mathcal{D}$ of $H$, there exists $S \in \mathcal{D}$ such that, for every $\mathcal{D}^{\prime} \subseteq \mathcal{D}$ with $S \in \mathcal{D}^{\prime}, \bigcup \mathcal{D}^{\prime}$ is either a k-hinge of $(V, E)$ or a subset of $E$ containing at most $k$ edges.

EXAMPLE 5.2. Consider a family of hypergraphs, $R_{n}, n \geq 2$. For every $n, R_{n}$ consists of $3 n$ points and $2 n+2$ edges. The points are labeled $x_{i}, y_{i}$, and $z_{i}$, $i=1, \ldots, n$, and the edges are as follows:

$$
\begin{aligned}
e_{x} & =\left\{x_{1}, \ldots, x_{n}\right\} \\
e_{y} & =\left\{y_{1}, \ldots, y_{n}\right\} \\
f_{i} & =\left\{x_{i}, y_{i}, z_{i}\right\}, i=1, \ldots, n ; \\
g_{i} & =\left\{z_{i}, x_{(i+1) \bmod n}, y_{(i+1) \bmod n}\right\}, i=1, \ldots, n .
\end{aligned}
$$



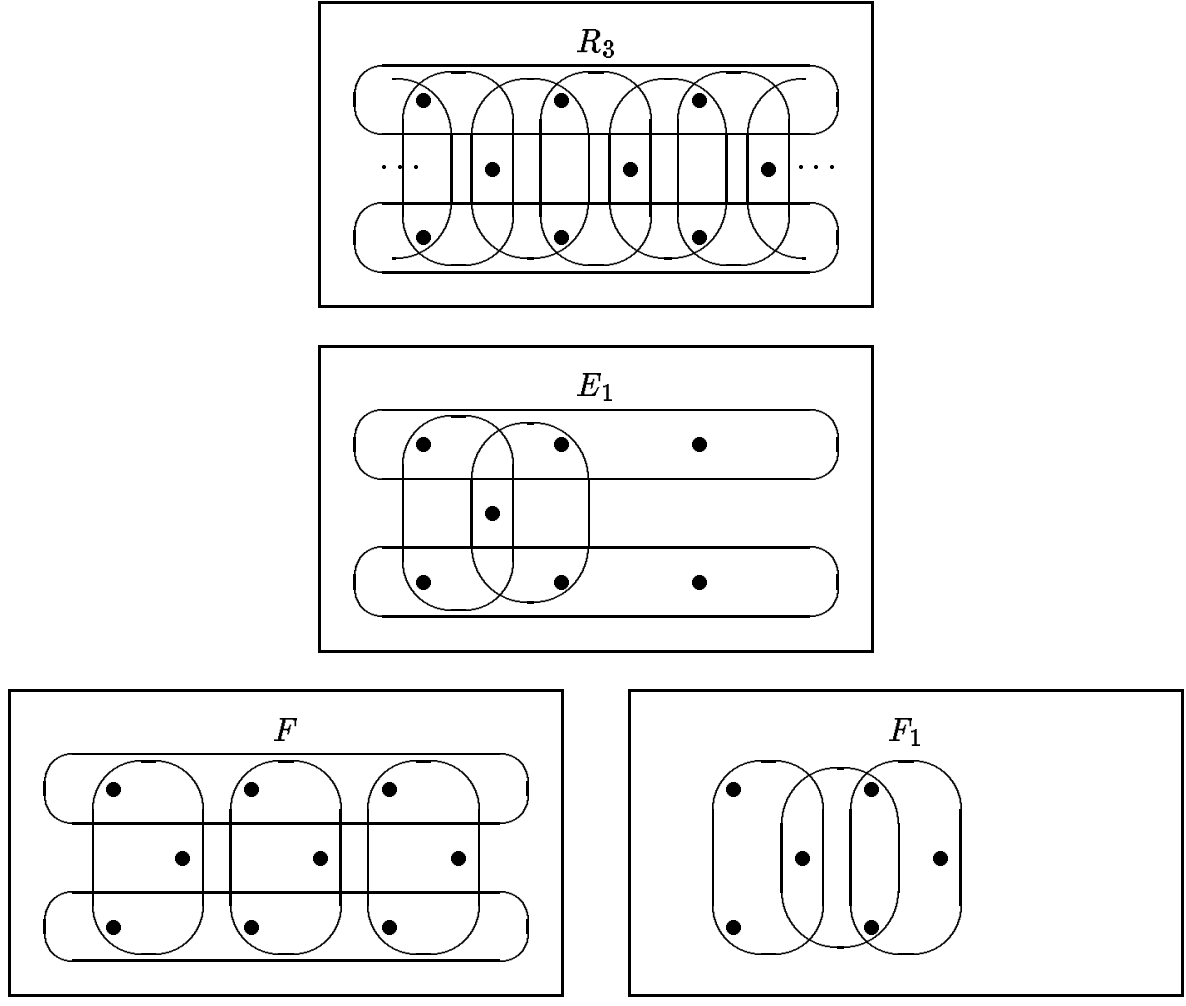

FiguRE 6 . The hypergraph $R_{3}$ and its minimal 2-hinges $E_{1}, F$, and $F_{1}$ (Example 5.2).

The minimal 2-hinges of $R_{n}$ are as follows:

$$
\begin{aligned}
E_{i} & =\left\{e_{x}, \epsilon_{y}, f_{i}, g_{i}\right\}, i=1, \ldots, n ; \\
F & =\left\{e_{x}, \epsilon_{y}, f_{1}, \ldots, f_{n}\right\} \\
G & =\left\{e_{x}, \epsilon_{y}, g_{1}, \ldots, g_{n}\right\} ; \\
F_{i} & =\left\{f_{i}, g_{i}, f_{(i+1) \bmod n}\right\}, i=1, \ldots, n ; \\
G_{i} & =\left\{g_{i}, f_{(i+1) \bmod n}, g_{(i+1) \bmod n}\right\}, i=1, \ldots, n .
\end{aligned}
$$

The hypergraph $R_{3}$ and its minimal 2-hinges $E_{1}, F$, and $F_{1}$ are illustrated in Figure 6 .

To see that 2-hinges do not have the Decomposition Property, consider the minimal 2-hinge $F$. Let $h=\left\{\epsilon_{x}, e_{y}\right\}$, and let $\mathcal{D}=\left\{\left\{e_{x}, e_{y}\right\},\left\{f_{1}\right\}, \ldots,\left\{f_{n}\right\}\right\}$. Clearly, $\mathcal{D}$ is an $h$-decomposition of $F$. Now notice that, for $i=1, \ldots, n$, $\left\{e_{x}, e_{y}, f_{i}\right\}$ is not a 2-hinge of $R_{n}$. Hence there is no $S \in \mathcal{D}$ such that every subset of $\mathcal{D}$ encompassing at least 3 edges including those of $S$ generates a 2-hinge of $(V, E)$.

As Proposition 3.1 and the Invariance Theorem rely on the Decomposition Property, we cannot hope to generalize their proofs to show the equivalent results 
for arbitrary $k$-hinges. As a matter of fact, a hypergraph can indeed have two different 2-hinge-trees where the size of the largest node is not the same, as the following example shows:

Example 5.3. Consider the family of hypergraphs $R_{n}, n \geq 2$, described in Example 5.2. A first possible 2-hinge-tree, $T_{E}$, is the linear list $E_{1}, \ldots, E_{n}$. A second possible 2-hinge-tree, $T_{F}$, is the star with central node $F$ and leaf nodes $F_{1}, \ldots F_{n}$. A third possible 2-hinge-tree, $T_{G}$, is the star with central node $G$ and leaf nodes $G_{1}, \ldots G_{n}$. The size of the largest node in $T_{E}$ is 4 , whereas the size of the largest node in both $T_{F}$ and $T_{G}$ is $n+2$.

A closer examination of the proof of Proposition 3.1 reveals that it does not rely on the full Decomposition Property, but rather on the consequent Inseparability Property. We might therefore attempt to remedy the situation by requiring that the nodes of a $k$-hinge-tree satisfy the Inseparability Property below, which is a generalization of the Inseparability Property for 1-hinges.

InSEPARABILITy Property FOR $k$-Hinges. Let $(V, E)$ be a hypergraph and let $H$ be a $k$-hinge of $(V, E)$. Then $H$ is inseparable if for every subset $h \subseteq E$ containing $k$ edges, and for every $h$-decomposition $\mathcal{D}$ of $H$, at most one element of $\mathcal{D}$ contains more than $k$ edges, and all elements of $\mathcal{D}$ except for a largest one contain at most $k$ edges in total.

Notice that, for general $k$-hinges, the Inseparability Property follows from the Decomposition Property in the same way as it does for 1-hinges. Furthermore notice that inseparability implies minimality:

Proposition 5.1. An inseparable $k$-hinge of a hypergraph is also minimal.

Proof. We prove the contrapositive. Thereto, let $(V, E)$ be a hypergraph and let $H$ be a $k$-hinge of $(V, E)$ that is not minimal. Thus $H$ contains another $k$-hinge of $(V, E)$, say $H^{\prime}$. Let $e$ be any edge of $H-H^{\prime}$, let $C_{e}$ be the connected component of $E-H^{\prime}$ with respect $H^{\prime}$ containing $e$ and let $h^{\prime}=\left\{\epsilon_{1}^{\prime}, \ldots, \epsilon_{k}^{\prime}\right\}$ be a corresponding separating edge set. Let $\mathcal{D}=\left\{\left\{e_{1}^{\prime}\right\}, \ldots,\left\{e_{k}^{\prime}\right\}, H \cap C_{e}, H-\right.$ $\left.\left(C_{e} \cup h^{\prime}\right)\right\}$. Clearly, $\mathcal{D}$ is an $h^{\prime}$-decomposition of $H$ which contains $k+2$ nonempty elements, whence it necessarily violates the second condition of the Inseparability Property for $k$-hinges. Hence $H$ is not inseparable.

On the other hand, not every minimal $k$-hinge is inseparable, as is shown by Example 5.4, below.

Example 5.4. Consider the following variation on Example 5.1. Let $\bar{G}$ be the hypergraph constructed from the graph $G$ in Figure 5 by adding a unique extra node to each of the edges $e_{1}, \ldots, e_{5}$, and by adding all binary edges connecting two extra nodes. Let $\bar{H}$ and $\bar{H}^{\prime}$ be the sets of edges of $\bar{G}$ corresponding to $H$ and $H^{\prime}$ in $G$, respectively. An exhaustive but straightforward examination of all subsets of $\bar{H}$ shows that $\bar{H}$ is a minimal 2-hinge. However, $\bar{H}$ is not inseparable, since $\left\{\bar{H}^{\prime}, \bar{H}-\bar{H}^{\prime}\right\}$ is an $\left\{e_{1}, e_{2}\right\}$-decomposition of $\bar{H}$ which does not satisfy the Inseparability Property for 2-hinges. 
Hence imposing the Inseparability Property on the nodes of a $k$-hinge-tree yields a genuine restriction.

We might hope that, with this restriction, it would be possible to derive an invariant for $k$-hinge-trees. Notice that Example 5.3 is not a counterexample to this proposal, as the nodes $F$ in $T_{F}$ and $G$ in $T_{G}$ are not inseparable, as can be easily verified. We conjecture that, using similar arguments as in the proof of Proposition 3.1, it is possible to find a bound for the largest inseparable $k$-hinge in terms of the size of the largest node in an arbitrary $k$-hinge-tree of which all the nodes are inseparable. Unfortunately, the bound will be weaker than in Proposition 3.1 and will not allow us to derive an equality as in the proof of the Invariance Theorem. In fact, Example 5.5 below demonstrates that the size of the largest node in a $k$-hinge-tree of which all the nodes are inseparable is not an invariant.

Example 5.5. Consider the hypergraph $G$ with 11 vertices, $v_{1}, \ldots, v_{11}$, and 7 edges, $e_{1}, e_{2}, e_{3}, f_{1}, \ldots, f_{4}$, defined as follows:

$$
\begin{array}{ll}
e_{1}=\left\{v_{1}, v_{2}, v_{3}, v_{4}\right\} ; & f_{1}=\left\{v_{1}, v_{3}, v_{4}, v_{8}, v_{9}\right\} \\
e_{2}=\left\{v_{8}, v_{9}, v_{10}, v_{11}\right\} ; & f_{2}=\left\{v_{2}, v_{3}, v_{4}, v_{10}, v_{11}\right\} \\
e_{3}=\left\{v_{4}, v_{5}, v_{6}, v_{7}\right\} ; & f_{3}=\left\{v_{4}, v_{5}, v_{7}, v_{8}, v_{10}\right\} \\
& f_{4}=\left\{v_{4}, v_{5}, v_{6}, v_{9}, v_{11}\right\}
\end{array}
$$

By an exhaustive search we find that the inseparable 2-hinges of $G$ are

$$
\begin{array}{lll}
\left\{e_{1}, e_{2}, e_{3}\right\} ; & \left\{\epsilon_{2}, e_{3}, f_{3}\right\} ; & \left\{e_{2}, f_{1}, f_{2}\right\} \\
\left\{e_{1}, e_{2}, f_{1}\right\} ; & \left\{\epsilon_{2}, e_{3}, f_{4}\right\} ; & \left\{e_{3}, f_{3}, f_{4}\right\} \\
\left\{e_{1}, e_{2}, f_{2}\right\} ; & \left\{\epsilon_{1}, f_{1}, f_{2}\right\} ; & \left\{f_{1}, f_{2}, f_{3}, f_{4}\right\}
\end{array}
$$

Two possible 2-hinge-trees of $G$ built from these inseparable 2-hinges are shown in Figure 5.5. The size of the largest node in $T_{\epsilon}$ is 3 , whereas the size of the largest node in $T_{f}$ is 4 .

The Hinge-Tree Decomposition Algorithm for 1-hinge-tree decompositions also relies on the Inheritance, Decomposition, and Inseparability Properties, so it cannot be directly generalized to $k$-hinges either. For example, the algorithm uses the fact that a 1-hinge-tree can be obtained by first decomposing the hypergraph into a tree of 1-hinges that are not necessarily minimal and then further decomposing these 1-hinges. As is shown in Example 5.1, a similar strategy for 2-hinge-trees is not guaranteed to yield a correct result. Another property the Hinge-Tree Decomposition Algorithm relies on is that a singleton separating edge set is always contained in some minimal 1-hinge. Unfortunately, this property does not carry over to 2-hinges, either. The following example exhibits a hypergraph having a separating edge pair not contained in any minimal 2-hinge of that hypergraph. 

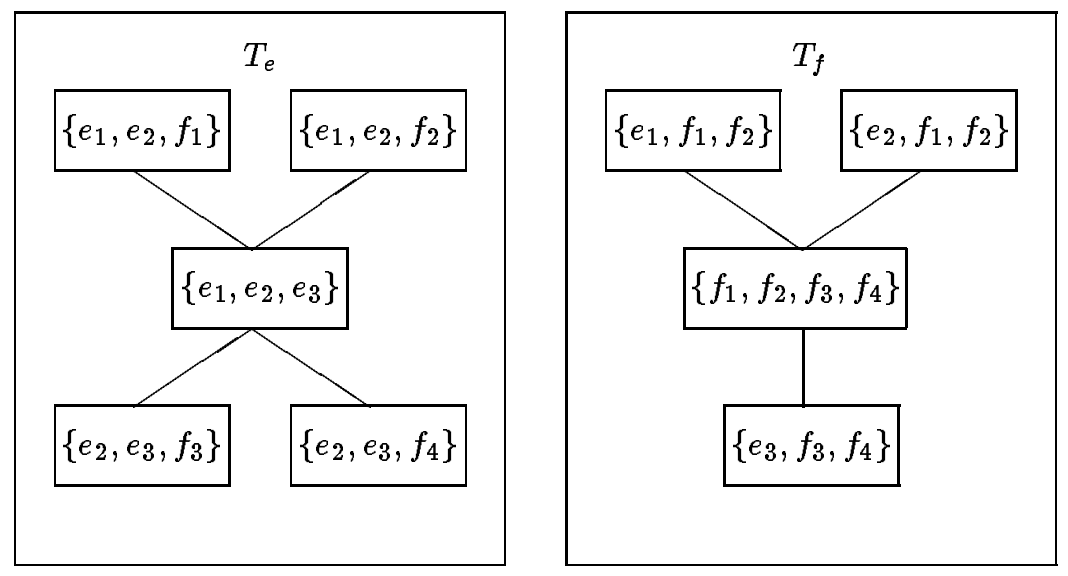

Figure 7. Two possible 2-hinge-trees for $G$ (Example 5.5).

Example 5.6. Consider the hypergraph with nodes

$$
\left\{x_{1}, x_{2}, y_{1}, y_{2}, z_{1}, z_{2}, u_{1}, u_{2}\right\}
$$

and edges

$$
\begin{array}{lll}
e_{1}=\left\{x_{1}, x_{2}, z_{1}, u_{1}\right\} ; & f_{1}=\left\{y_{1}, y_{2}, z_{1}, u_{2}\right\} ; & g_{1}=\left\{x_{1}, y_{1}\right\} \\
e_{2}=\left\{x_{1}, x_{2}, z_{2}, u_{2}\right\} ; & f_{2}=\left\{y_{1}, y_{2}, z_{2}, u_{1}\right\} ; & g_{2}=\left\{x_{2}, y_{2}\right\}
\end{array}
$$

Clearly, $\left\{g_{1}, g_{2}\right\}$ is a separating edge pair separating off $\left\{e_{1}, f_{1}\right\}$ from $\left\{e_{2}, f_{2}\right\}$. Now, the smallest 2-hinges (with respect to inclusion) containing $\left\{g_{1}, g_{2}\right\}$ are the edge sets $\left\{e_{1}, f_{1}, g_{1}, g_{2}\right\},\left\{\epsilon_{2}, f_{2}, g_{1}, g_{2}\right\},\left\{e_{1}, f_{2}, g_{1}, g_{2}\right\}$, and $\left\{e_{2}, f_{1}, g_{1}, g_{2}\right\}$, as can easily be verified by a simple, exhaustive search. None of these four 2-hinges are minimal however, as all eight sets obtained from them by either removing $g_{1}$ or $g_{2}$ are 2-hinges, too.

The above examples clearly show that an algorithm for generating $k$-hingetrees cannot simply be obtained by a straightforward generalization of the HingeTree Decomposition Algorithm. As a matter of fact, it is an open problem whether or not there exists an efficient and effective algorithm for finding $k$ hinge-trees.

\section{Conclusions}

We have shown that any hypergraph has a decomposition into a tree of 1hinges, and the size of the largest minimal hinge in such a tree is an invariant of the hypergraph. This provides a classification scheme for hypergraphs giving a useful measure of their structural complexity.

The extension of the notion of a 1-hinge to $k$-hinges allows more refined decompositions in many cases, but $k$-hinges fail to have the pertinent properties of inheritance and inseparability. Without inheritance it is difficult to guarantee 
the existence of a tree decomposition into minimal $k$-hinges, whence the existence of an invariant on the structure of $k$-hinges is unlikely. Even when trees do exist, it is not clear whether there is a general algorithm to construct them.

One approach to these problems is to strengthen the definition of a minimal $k$-hinge in order to guarantee the required properties. Alternatively, it may be necessary to formulate a more sophisticated definition of the invariant.

In the light of these results, it is clear that the degree of cyclicity is a strikingly simple and important measure for the structural complexity of a hypergraph.

\section{REFERENCES}

1. S. Arnborg, Efficient algorithms for combinatorial problems on graphs with bounded decomposability-a survey. BIT 25 (1985), 2-23.

2. C. Beeri et al., Properties of acyclic database schemes. Proc. 13th Annual ACM Symposium on the Theory of Computing (Milwaukee, 1981), ACM Press, New York, pp. 355-362.

3. C. Beeri et al., On the desirability of acyclic database schemes. J. Assoc. Comput. Mach. 30 (1983), 479-513.

4. C. Bergé, Graphs and Hypergraphs, North-Holland, New York, 1976.

5. B. Courcelle, Graph rewriting: an algebraic and logic approach. Handbook of Theoretical Computer Science (J. van Leeuwen, ed.), Elsevier Science Publishers, Amsterdam, 1990, p. 224.

6. E. C. Freuder, A sufficient condition for backtrack-bounded search. J. Assoc. Comput. Mach. 32 (1985), 755-761.

7. M. Gyssens, On the complexity of join dependencies. ACM Trans. Database Systems 11 (1986), 81-108.

8. M. Gyssens, P. Jeavons, and D. Cohen, Decomposing constraint satisfaction problems using database techniques. Artificial Intelligence 66 (1994), 57-89.

9. M. Gyssens and J. Paredaens, A decomposition methodology for cyclic databases. Advances in Database Theory, vol. 2 (Toulouse, 1983, H. Gallaire, J. Minker, and J. M. Nicolas, eds.), Plenum Press, New York, 1984, pp. 85-122.

10. On the decomposition of join dependencies. Proc. 3rd ACM SIGACT-SIGMOD Symposium on Principles of Database Systems (Waterloo, Ont., 1984), ACM Press, New York, 1984, pp. 143-152.

11. Yannakakis, M., Computing the minimum fill-in is NP-complete. SIAM J. Algebraic Discrete Methods 2 (1981), 77-79.

Department of Computer Science, Royal Holloway, University of London, Egham, SURREY TW20 0EX, ENGLAND

E-mail address: pete@dcs.rhbnc.ac.uk

Department of Computer Science, Royal Holloway, University of London, Egham, SuRREY TW20 0EX, England

E-mail address: pete@dcs.rhbnc.ac.uk

Department WNi, University of Limburg (LUC), B-3590 Diepenbeek, Belgium

E-mail address: gyssens@charlie.luc.ac.be 